\title{
Mudança na biomecânica da postura sentada afeta a função pulmonar
}

\author{
Change in biomechanics of sitting posture affects the pulmonary function \\ Alteración en la biomecánica de la postura sentada afecta la función pulmonar \\ Adriana Maria Contesini', Thiago Henrique da Silva², Francis Meire Favero³, Silvana Maria Blascovi-Assis ${ }^{4}$, \\ Mariana Callil Voos 5 , Fátima Aparecida Caromano 6
}

RESUMO | Este trabalho teve como objetivo caracterizar as posturas induzidas por dois sistemas diferentes de cadeiramesa e analisar seus efeitos na função pulmonar. Trata-se de estudo transversal, descritivo, do tipo sujeito único e intrasséries (A-B, B-A), com coleta em dias consecutivos. Participaram da pesquisa 15 voluntárias e foram utilizados dois sistemas cadeira-mesa: convencional (A) e experimental (B). A postura foi avaliada por meio de fotogrametria em cada um dos sistemas, com imagens analisadas por meio do programa AutoCAD 2010. Posteriormente, foram calculados os ângulos articulares da postura média das participantes em cada sistema. Os dados posturais e respiratórios foram comparados considerando as diferentes posições adotadas. O sistema cadeira-mesa convencional promoveu dois diferentes padrões posturais: um deles apresentou ângulos articulares similares aos do sistema experimental, com resultados de espirometria semelhantes, e o outro padrão apresentou ângulos corporais de acordo com os padrões esperados com valores de espirometria significativamente inferiores em $V E F_{1}$, $\mathrm{VEF}_{1} / \mathrm{CVF}$ e $\mathrm{FEF}_{\text {máx }}$ O sistema experimental diferiu de valores de espirometria da postura ortostática relatados na literatura somente em $\mathrm{FEF}_{\text {máx }}$, sugerindo similaridade de condição postural. Conclui-se que o mobiliário experimental melhorou a função respiratória na postura sentada em comparação com o mobiliário tradicional, podendo beneficiar pessoas em condições especiais, como gestantes, obesos e pessoas com doenças pulmonares crônicas.

Descritores | Postura; Mecânica Respiratória; Espirometria; Ergonomia.
ABSTRACT I The objective of this study was to characterize the postures induced by two different chair-desk systems and analyze their effects on lung function. This was a crosssectional, descriptive study of single subject with intraseries type analysis ( $A-B, B-A$ ) during consecutive days of data collection. Fifteen volunteers participated using two chair-desk systems: conventional (A) and experimental (B). Postural evaluation was performed in both systems using photogrammetry. These images were analyzed using AutoCAD 2010, estimating the average position of the joint angles of individuals in each system. These values were analyzed verifying the averages in each posture. Postural and respiratory data were compared by checking whether the different positions adopted by the participants resulted in changes in the spirometry values. Conventional chair-desk system promoted two different postural patterns, considering that one presented joint angles similar to experimental system, with similar spirometry results and the other presented body angles according to the reference of standards and spirometry results significantly lower in $\mathrm{FEV}_{1}, \mathrm{FEV}_{1} / \mathrm{FVC}$ and $\mathrm{FEF}_{\text {max }}$. Experimental system differed from values of literature in standing posture only in $\mathrm{FEF}_{\text {max }}$, suggesting similarity of postural situation. It was concluded that the experimental furniture proved a tool capable of benefiting respiratory function in sitting posture and may be an option to benefit people in special conditions such as pregnant women, obese individuals and people with chronic pulmonary diseases.

Keywords I Posture; Respiratory Mechanics; Spirometry; Ergonomics. 
RESUMEN | Este estudio tuvo como objetivo caracterizar las posturas inducidas por dos sistemas diferentes de silla-mesa y analizar sus efectos sobre la función pulmonar. Se trata de un estudio transversal, descriptivo, de tipo de un solo sujeto e intraseries ( $A-B, B-A$ ), con recolección en días consecutivos. Quince voluntarios participaron en el estudio, y se utilizaron dos sistemas de silla-mesa: convencional (A) y experimental (B). La evaluación postural se realizó mediante fotogrametría en cada uno de los sistemas, con imágenes analizadas por medio del programa AutoCAD 2010. Posteriormente, se calcularon los ángulos de articulación de la postura media de las participantes en cada sistema. Los datos posturales y respiratorios se compararon considerando las diferentes posiciones adoptadas. El sistema de silla-mesa convencional promovió dos patrones posturales diferentes: uno presentó ángulos de articulación similares al sistema experimental, con resultados de espirometría similares, y el otro estándar presentó ángulos corporales de acuerdo con los patrones esperados con resultados de espirometría significativamente más bajos en $V E F_{1}, V_{1} F_{1} / C V F$ y $F_{E F}$ max El sistema experimental difería de los valores de espirometría de la postura ortostática informados en la literatura solo en $\mathrm{FEF}_{\text {max }}$ lo que sugiere una similitud de la condición postural. Se concluyó que los muebles experimentales pueden mejorar la función respiratoria en la posición sentada cuando se comparaban con los muebles tradicionales, y pueden beneficiar a personas en condiciones especiales, como mujeres embarazadas, personas obesas y personas con enfermedades pulmonares crónicas.

Palabras clave I Postura; Mecánica Respiratoria; Espirometría; Ergonomía.

\section{INTRODUÇÃO}

A postura sentada é a mais frequentemente adotada pelo homem ${ }^{1}$, sendo induzida pelo mobiliário utilizado, que leva à adoção de determinados padrões posturais ${ }^{2}$. Assim, há influência direta desta postura nos sistemas musculoesquelético ${ }^{3}$, respiratório ${ }^{4}$, circulatório ${ }^{5}$ e metabólico ${ }^{6}$, podendo comprometer de maneira negativa o organismo humano quando relacionada com o sedentarismo ${ }^{7}$.

O tempo de permanência na postura sentada gerou recentemente maior interesse entre pesquisadores de saúde, empregadores e funcionários, além de atrair atenção significativa dos meios de comunicação ${ }^{8}$. Diretrizes de saúde recentes, reconhecidas internacionalmente, recomendam que adultos diminuam a quantidade diária de permanência nesta postura, pois esta aumenta os riscos relacionados ao sedentarismo ${ }^{9,10}$. Afeta de maneira significativa, a mecânica ventilatória humana, e quando não há apoio nas costas a ativação dos músculos respiratórios é maior, resultando no aumento do volume corrente ${ }^{11}$. Em relação à postura sentada inclinada, outros aspectos também se mostram desiguais, como a inalação de partículas, possivelmente pelas mudanças na distribuição regional da ventilação entre estas posturas ${ }^{4}$.

O tipo de cadeira utilizada afeta o suporte postural, a atividade muscular, o alívio da pressão intradiscal, a função pulmonar, a mobilidade e o conforto ${ }^{12}$. Na postura sentada considerada ideal para os padrões ocidentais, a pessoa deve manter os quadris fletidos em aproximadamente $90^{\circ}$, o que leva a uma retificação ou inversão da curva lombar², ao deslocamento ântero-superior do conteúdo abdominal e à diminuição da posição ideal da musculatura abdominal e diafragmática, reduzindo sua capacidade de contração ${ }^{13}$. Em função disto, ocorre adaptação da função respiratória a esta postura, que é afetada pelo tipo de apoio para as costas, podendo gerar uma discreta restrição à expansão torácica ${ }^{13,14}$.

Como diferentes tipos de mobiliários podem causar variações da função respiratória, o uso de tipos diferenciados pode minimizar eventuais efeitos deletérios da postura sentada no mecanismo ventilatório. Poucos são os estudos encontrados sobre este assunto. Assim, faz-se necessário avaliar parâmetros variados de postura sentada e sua relação com a respiração.

O objetivo deste estudo foi caracterizar as posturas induzidas por dois sistemas cadeira-mesa diferentes e analisar os efeitos destas posturas na função pulmonar.

\section{METODOLOGIA}

\section{Participantes e considerações éticas}

Participaram do estudo 15 voluntárias do sexo feminino, numa amostra de conveniência. Os critérios de inclusão foram: estudantes universitárias, regularmente matriculadas em uma universidade pública, caucasianas, destras, saudáveis, sedentárias, com índice de massa corporal (IMC) entre $18,5 \mathrm{~kg} / \mathrm{m}^{2}$ a $24,9 \mathrm{~kg} /\left(\mathrm{m}^{2}\right)^{15}$. A escolha da população caucasiana se relacionou às diferenças nos padrões da postura sentada decorrentes 
de hábitos culturais ${ }^{1}$ e de possíveis variações na estrutura corporal por diferenças étnicas ${ }^{16}$. A decisão de avaliar exclusivamente mulheres considerou as diferenças anatomofisiológicas entre os sexos que poderiam afetar os resultados.

As participantes foram informadas sobre os procedimentos relativos ao estudo e assinaram o Termo de Consentimento Livre e Esclarecido.

\section{Análise estatística}

O poder da amostra foi calculado pelo programa GPower 3.0. O cálculo do tamanho da amostra foi baseado em duas variáveis (capacidade vital forçada e ângulo de flexão da pelve durante a postura sentada), considerando o delineamento estatístico do teste F para medidas repetidas (efeito entre e dentro dos grupos), com tamanho do efeito moderado $(\mathrm{f}=0,3)$, nível de significância estatístico de $80 \%$ e erro alfa de $5 \%$, o que resultou em $n=15$. Os dados coletados foram tratados com análise estatística descritiva e teste t de Student para comparação entre grupos.

\section{Mobiliários utilizados}

Foram escolhidos dois sistemas cadeira-mesa diferentes, especialmente pela indução do ângulo do quadril. $\mathrm{O}$ mobiliário convencional (sistema A) correspondeu ao conceito clássico de sistema cadeira-mesa padrão para a permanência na postura sentada, cuja cadeira induz a um ângulo de aproximadamente $90^{\circ}$ do quadril e joelhos ${ }^{1,2,17}$. A mesa utilizada apresentava tampo em $90^{\circ}$ com a vertical.

No mobiliário experimental (sistema B), a cadeira experimental (kneeling chair) foi escolhida por preservar a curvatura lombar, aumentando o ângulo de flexão do quadril $^{18}$, e a mesa apresentava inclinação do tampo em $20^{\circ}$, por oferecer maior conforto visual, induzir menor flexão cervical e proporcionar melhor apoio para os antebraços ${ }^{19}$.

No sistema $A$, a mesa de madeira possuía um metro de altura, superfície de $1,00 \mathrm{~m} \times 0,80 \mathrm{~m}$, tampo alinhado com a horizontal. A cadeira apresentava altura ajustável, assento de $30 \mathrm{~cm} \times 30 \mathrm{~cm}$, a $0^{\circ}$ em relação à horizontal e apoio lombar regulável em altura. No sistema B o tampo da mesa apresentava inclinação de $20^{\circ}$ e a cadeira tinha $50 \mathrm{~cm}$ de altura em relação ao solo, assento de $33 \times 43 \mathrm{~cm}$ e inclinação de $30^{\circ}$ em relação à horizontal, apoio para os joelhos com $30 \mathrm{~cm} \times 43 \mathrm{~cm}$ e inclinação de $25^{\circ} \mathrm{em}$ relação à horizontal.
A sequência de utilização dos mobiliários foi definida por sorteio por cada participante. Foram elaborados dois envelopes, sendo que a sequência 1 correspondia à seguinte ordem: $\mathrm{A}-\mathrm{B}, \mathrm{B}-\mathrm{A}, \mathrm{A}-\mathrm{B}, \mathrm{B}-\mathrm{A}, \mathrm{A}-\mathrm{B}$ e a sequência 2 à ordem: $\mathrm{B}-\mathrm{A}, \mathrm{A}-\mathrm{B}, \mathrm{B}-\mathrm{A}, \mathrm{A}-\mathrm{B}, \mathrm{B}-\mathrm{A}$. As participantes tiveram contato prévio individual com cada um dos mobiliários utilizados neste experimento pelo período de uma hora, um mês antes do início da coleta de dados para o reconhecimento dos mesmos e, durante o experimento, foram orientadas a assumir a postura de maior conforto em cada mobiliário, de forma a não induzir posturas específicas.

O tempo de permanência em cada mobiliário foi determinado com base num estudo de Corlett e Bishop ${ }^{20}$, segundo o qual a manutenção de uma postura inadequada pode durar, no máximo, de 1 a 5 minutos, até que comecem a aparecer dores. Como este estudo não visava analisar o conforto do mobiliário, optou-se por um período intermediário de três minutos.

\section{Coleta de dados}

As participantes foram divididas em três grupos, sendo que cada grupo compareceu por cinco dias consecutivos para a realização das avaliações.

Durante estes cinco dias, entre nove e 11 horas da manhã, as participantes foram recebidas individualmente em uma sala de $4 \times 6 \mathrm{~m}$, com iluminação controlada e temperatura constante a $26^{\circ}$, vestidas em roupa de banho e com cabelos presos.

Foram realizadas medidas de altura e peso usando balança clínica marca Filizzolla ${ }^{\circledR}$, e a marcação, por meio de palpação, dos pontos anatômicos a serem avaliados utilizou referências citadas no tutorial do programa Software para Avaliação Postural (Sapo) ${ }^{21,22}$, sendo utilizados os pontos a seguir: trago, $\mathrm{C}$, acrômio, epicôndilo lateral, porção distal da ulna, trocânter maior do fêmur, linha articular do joelho e maléolo lateral, que foram marcados com adesivos e bolas de isopor de $1 \mathrm{~cm}$ no hemicorpo direito ${ }^{22,23}$.

$\mathrm{Na}$ sequência, as participantes permaneceram em repouso em decúbito dorsal ${ }^{24}$ por cinco minutos, de acordo com o preconizado no Consenso Brasileiro de Espirometria $^{25}$, ao final dos quais realizaram uma prova de função pulmonar na posição ortostática, utilizando um espirômetro Microquark, marca Cosmed ${ }^{\circledR}$. O exame em postura ortostática foi realizado para eliminar a possibilidade de qualquer intercorrência clínica respiratória no dia do experimento, e posteriormente utilizado como parâmetro de comparação de dados. 
A seguir, as participantes sentaram no mobiliário sorteado, descansaram por cinco minutos, realizaram nova prova de função pulmonar e realizaram uma atividade gráfica por três minutos ${ }^{26}$. Ao final deste período foi realizado novo teste respiratório.

Esta atividade gráfica, baseada na reprodução de desenhos geométricos simples, como quadrados e triângulos, foi elaborada especificamente para este estudo, baseando-se em testes psicológicos de reprodução de figuras geométricas, com nível de complexidade crescente ${ }^{26}$. Neste caso, buscou-se manter a atenção da participante nesta atividade e não na postura/mobiliário. Após novo descanso de cinco minutos o procedimento foi repetido no segundo mobiliário, conforme sorteado. As participantes foram, então, dispensadas para retornar no dia seguinte, no mesmo horário. Estes procedimentos foram aplicados por uma única pesquisadora, previamente treinada.

A coleta das imagens consistiu na filmagem das participantes em vista lateral direita utilizando-se câmera digital Canon PowerShot ${ }^{\circledR}$ SX30 IS 14,1 megapixels, com capacidade de gravação de 30 quadros por segundo, posicionada paralela ao chão por meio de tripé nivelado a um metro e meio de altura e a quatro metros de distância do mobiliário utilizado, ambos alinhados por meio de nível. Desta forma, a distância e altura da máquina fotográfica em relação ao sujeito de pesquisa foram determinadas considerando-se as características físicas do local de coleta, a iluminação e as características técnicas do equipamento. Especificamente neste estudo, o protocolo proposto pelo método de avaliação postural Sapo não permite boa observação do mobiliário, posto que não foi elaborado para esta finalidade.
A referência vertical foi obtida a partir de um fio de prumo fixado no teto, suspenso a uma distância de um metro do ângulo póstero-lateral direito da mesa, posteriormente à cadeira.

Após as filmagens, foram selecionadas fotografias por quadros de cada gravação por meio do programa Real Media Player Basic, opção "fazer montagem", que permite salvar imagens em formato JPEG. O primeiro quadro correspondeu à posição inicial do indivíduo e, a seguir, foi capturado um quadro a cada cinco segundos, totalizando 38 quadros por experimento, 76 quadros por dia e 380 quadros por participante, num total de 5.700 fotografias obtidas no experimento completo.

A análise das imagens foi realizada utilizando-se o programa AutoCAD ${ }^{\circledR} 2010$ para a mensuração dos ângulos relativos aos pontos anatômicos marcados previamente ${ }^{27}$. Estes ângulos foram tabelados utilizando-se o programa Excel $^{\circledR}$ 2010, estimando-se sua média simples e desvio padrão e determinando-se a postura média dos indivíduos em cada mobiliário.

Com base nestes dados, foi elaborado, por meio do programa Régua e Compasso, um diagrama caracterizando a postura mais frequente de cada sujeito, para cada um dos mobiliários, de acordo com a postura média do grupo, com o objetivo exclusivo de facilitar a visualização dos padrões encontrados (Figura 1). A Comparação de dados entre grupos utilizou o teste de Wilcoxon e o t de Student.

A coleta destes dados foi realizada por um técnico em informática, especialista em AutoCAD ${ }^{\circledR}$ e usuário do programa Régua e Compasso.
Fio de prumo

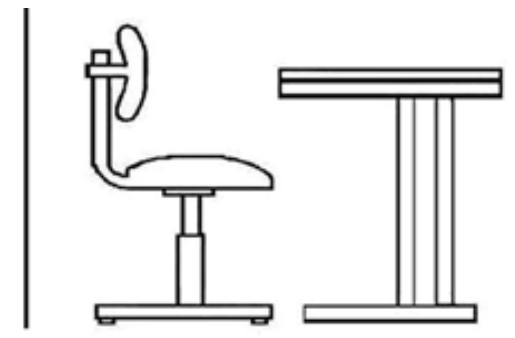

Sistema A

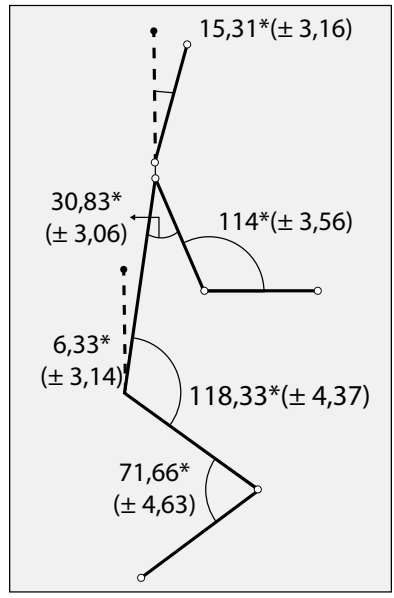

Subgroupo A1

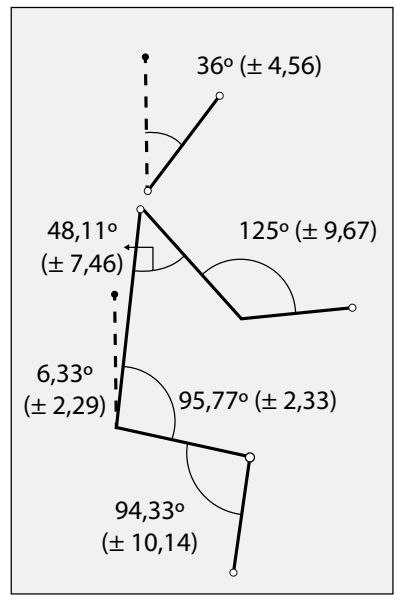

Subgroupo A2 
Em relação à avaliação pulmonar, os exames seguiram a padronização internacional, medindo a capacidade vital forçada $(\mathrm{CVF})$, volume expiratório forçado no primeiro segundo $\left(\mathrm{VEF}_{1}\right)$, relação $\mathrm{VEF}_{1} / \mathrm{CVF}$, fluxo expiratório forçado entre $25 \%$ e $75 \%$ da CVF (FEF25-75\%) e fluxo expiratório máximo $\left(\mathrm{FEF}_{\text {máx }}\right)^{28}$.

Esta avaliação foi realizada por médico pneumologista, especializado em prova de função pulmonar, cego para o estudo.

Os valores encontrados foram analisados a partir do cálculo das médias obtidas em cada postura. Os dados posturais e respiratórios foram submetidos à análise para verificação de relações entre as diferentes posições adotadas pelas participantes, se resultaram em alterações nos valores espirométricos.

\section{RESULTADOS}

\section{Caracterização das participantes}

As participantes foram caracterizadas de acordo com as variáveis: idade, peso e altura, sendo a idade média de 19,3 31,9 anos (18 a 22 anos), a altura média de $162 \pm 2,3 \mathrm{~cm}(149 \mathrm{~cm} \mathrm{a}$ $174 \mathrm{~cm})$ e o peso médio de $59,61 \pm 4,85 \mathrm{~kg}(52,7 \mathrm{~kg}$ a $67,5 \mathrm{~kg})$.

Em relação à sequência de utilização dos mobiliários, as participantes $4,6,8,9,13,14$ e 15 realizaram a sequência 1 e as participantes $1,2,3,5,7,10,11$ e 12 , executaram a sequência 2 .

\section{Características posturais promovidas pelos dois mobiliários}

Em relação à coleta dos dados dos ângulos posturais, foi realizada análise estatística utilizando-se os programas Excel 2010, Minitab v.14 e Statistica v.8. Foi testada a normalidade de cada variável por meio do teste Kolmogorov-Smirnov e, verificada a normalidade, realizados os testes de Barlett e Levene para confirmar sua homogeneidade.

Foi elaborado um diagrama baseado nos ângulos posturais (médias e desvios-padrões) promovidos pelos sistemas A e B, representando a postura média apresentada pelas integrantes de cada grupo. Analisando estes diagramas encontrou-se, no sistema $\mathrm{A}$, dois subgrupos com padrões posturais distintos, A1 e A2 (Figura 2).
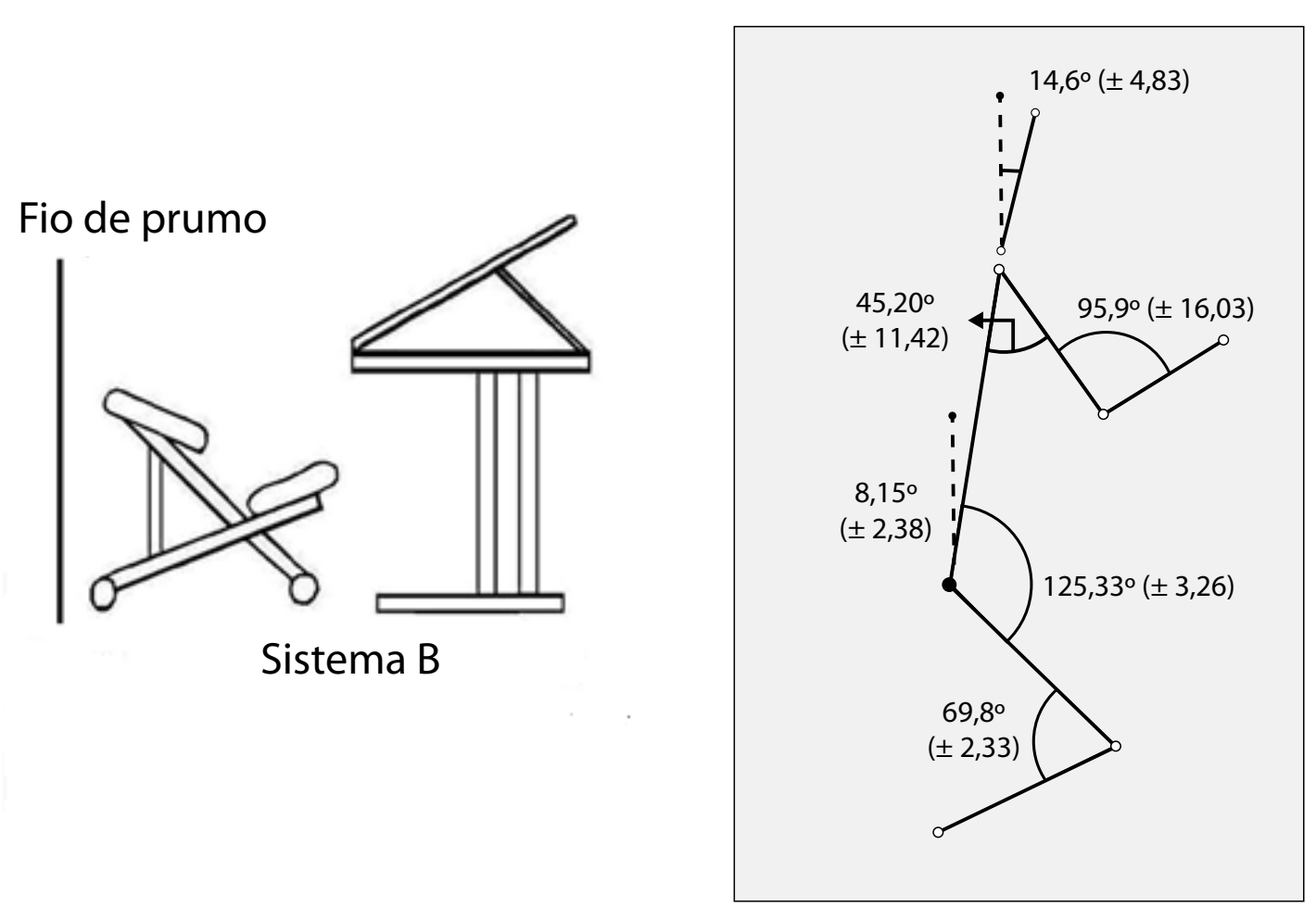

Figura 2. Postura induzida pelo sistema cadeira-mesa experimental (sistema B)

O subgrupo A1 ( $\mathrm{n}=6$ ) foi composto pelas participantes 2, $3,8,12,13,14$ e o subgrupo $A 2(n=9)$ pelas participantes 1 , 4 , $5,6,7,9,10,11$ e 15 . No subgrupo A2, a postura apresentou ângulos de flexão de quadril e joelho com valores próximos aos considerados como ideais para a postura sentada (cerca de $90^{\circ}$ ), mas com uma acentuada anteriorização da cabeça.

No sistema B observou-se um padrão postural semelhante em todas as participantes. A postura 
encontrada no sistema $\mathrm{B}$ foi semelhante à do subgrupo A1, caracterizando-se por ângulo de flexão de quadril mais aberto (maior que $90^{\circ}$ ), ângulo de flexão de joelho mais fechado (menor que $90^{\circ}$ ) e menor anteriorização da cabeça.

\section{Características da função respiratória nas diferentes posturas encontradas}

Para as avaliações por espirometria foram utilizados o teste de Wilcoxon e o teste $t$ de Student para amostras pareadas, comparando a posição ortostática com cada um dos mobiliários avaliados, os dois mobiliários entre si, e os dois subgrupos encontrados no mobiliário convencional. Foram aceitos como resultados significantes aqueles com $\mathrm{p} \leq 0,05$ (Tabela 1 ).

Os dados obtidos na avaliação pulmonar para cada mobiliário, em cada um dos cinco dias, não apresentaram diferenças estatisticamente significantes para nenhuma das 15 participantes, como esperado. Assim, trabalhou-se com a média dos valores individuais para cada variável em cada mobiliário.

Tabela 1. Comparação dos índices espirométricos entre a posição ortostática e os sistemas A e B, sistema A x sistema B e subgrupo A1 x subgrupo A2

\begin{tabular}{|c|c|c|c|c|c|c|c|c|c|c|c|c|}
\hline \multirow{2}{*}{$\begin{array}{l}\text { Variável } \\
\text { CVF (L) }\end{array}$} & \multicolumn{2}{|c|}{$\begin{array}{c}\text { Sistema A x } \\
\text { Bipedestação }\end{array}$} & \multirow{2}{*}{$\begin{array}{c}\text { p-valor } \\
0,91\end{array}$} & \multicolumn{2}{|c|}{$\begin{array}{c}\text { Sistema B x } \\
\text { Bipedestação }\end{array}$} & \multirow{2}{*}{$\begin{array}{c}\text { p-valor } \\
0,36\end{array}$} & \multicolumn{2}{|c|}{$\begin{array}{l}\text { Sistema A X } \\
\text { Sistema B }\end{array}$} & \multirow{2}{*}{$\begin{array}{c}\text { p-valor } \\
0,45\end{array}$} & \multicolumn{2}{|c|}{$\begin{array}{c}\text { Subgrupo A1 x } \\
\text { Subgrupo A2 }\end{array}$} & \multirow{2}{*}{$\begin{array}{c}\text { p-valor } \\
0,49\end{array}$} \\
\hline & $\begin{array}{c}4,25 \\
\pm 0,88\end{array}$ & $\begin{array}{c}4,27 \\
\pm 0,89\end{array}$ & & $\begin{array}{c}4,29 \\
\pm 0,87\end{array}$ & $\begin{array}{c}4,27 \\
\pm 0,89\end{array}$ & & $\begin{array}{c}4,25 \\
\pm 0,88\end{array}$ & $\begin{array}{c}4,29 \\
\pm 0,87\end{array}$ & & $\begin{array}{c}4,29 \\
\pm 0,54\end{array}$ & $\begin{array}{c}4,23 \\
\pm 0,57\end{array}$ & \\
\hline VEF1 (L) & $\begin{array}{c}3,67 \\
\pm 0,87\end{array}$ & $\begin{array}{c}3,69 \\
\pm 0,90\end{array}$ & $<0,01$ & $\begin{array}{c}3,69 \\
\pm 0,80\end{array}$ & $\begin{array}{c}3,69 \\
\pm 0,90\end{array}$ & 0,28 & $\begin{array}{c}3,67 \\
\pm 0,87\end{array}$ & $\begin{array}{c}3,69 \\
\pm 0,80\end{array}$ & $<0,01$ & $\begin{array}{c}3,71 \\
\pm 0,78\end{array}$ & $\begin{array}{r}3,65 \\
\pm 0,97\end{array}$ & $<0,01$ \\
\hline VEF1/CVF (\%) & $\begin{array}{l}85,01 \\
\pm 5,2\end{array}$ & $\begin{array}{l}86,11 \\
\pm 5,2\end{array}$ & $<0,01$ & $\begin{array}{l}86,11 \\
\pm 5,2\end{array}$ & $\begin{array}{l}86,11 \\
\pm 5,2\end{array}$ & 0,09 & $\begin{array}{l}85,01 \\
\pm 5,2\end{array}$ & $\begin{array}{l}86,11 \\
\pm 5,2\end{array}$ & $<0,01$ & $\begin{array}{l}86,15 \\
\pm 5,5\end{array}$ & $\begin{array}{l}85,12 \\
\pm 5,6\end{array}$ & $<0,01$ \\
\hline FEF 25-75 (L/s) & $\begin{array}{r}4,28 \\
\pm 1,22\end{array}$ & $\begin{array}{r}4,3 \\
\pm 1,2\end{array}$ & 0,55 & $\begin{array}{l}4,32 \\
\pm 1,8\end{array}$ & $\begin{array}{r}4,3 \\
\pm 1,2\end{array}$ & 0,55 & $\begin{array}{r}4,28 \\
\pm 1,22\end{array}$ & $\begin{array}{l}4,32 \\
\pm 1,8\end{array}$ & 0,26 & $\begin{array}{r}4,35 \\
\pm 1,62\end{array}$ & $\begin{array}{c}4,2 \\
\pm 1,25\end{array}$ & 0,28 \\
\hline FEF máx. (L/s) & $\begin{array}{l}7,99 \\
\pm 2,21\end{array}$ & $\begin{array}{c}8,6 \\
\pm 2,42\end{array}$ & $<0,01$ & $\begin{array}{c}8,54 \\
\pm 2,38\end{array}$ & $\begin{array}{c}8,6 \\
\pm 2,42\end{array}$ & $<0,01$ & $\begin{array}{l}7,99 \\
\pm 2,21\end{array}$ & $\begin{array}{r}8,54 \\
\pm 2,38\end{array}$ & $<0,01$ & $\begin{array}{r}8,34 \\
\pm 2,33\end{array}$ & $\begin{array}{l}7,97 \\
\pm 2,3\end{array}$ & $<0,01$ \\
\hline
\end{tabular}

*CVF: capacidade vital forçada; VEF;: volume expiratório forçado no primeiro segundo; FEF: fluxo expiratório médio (25-75\%) obtido na manobra de CVF; FEF Max: Fluxo expiratório máximo durante a manobra de CVF; e L/s: litros por segundo.

Considerando a postura sentada no sistema A e a posição ortostática, foi observado diferença significativa com aumento em $\mathrm{VEF}_{1}, \mathrm{VEF}_{1} / \mathrm{CVF}_{0}$ e $\mathrm{FEF}_{\text {máx }}$ no ortostatismo em relação ao mobiliário tradicional, enquanto a CVF e o FEF 25-75\% não mostraram diferenças estatísticas entre as duas condições posturais.

Em relação à situação experimental comparada com o ortostatismo, foi observado alteração significativa somente em $\mathrm{FEF}_{\text {máx }}$, sendo este maior na posição ortostática.

Comparando os dados da posição sentada experimental e convencional observou-se que, com exceção da CVF e do FEF 25-75\%, os demais parâmetros apresentaram redução estatisticamente significativa na posição convencional.

$\mathrm{Na}$ comparação entre os dois subgrupos encontrados no mobiliário convencional, foram observadas diferenças estatisticamente significativas na $\mathrm{VEF}_{1}, \mathrm{VEF}_{1} / \mathrm{CVF} \%$ e $\mathrm{FEF}_{\text {máx }}$, com o subgrupo A1 apresentando valores mais altos que o subgrupo A2.

As alterações dos parâmetros espirométricos em diferentes condições posturais foram replicáveis nos dois mobiliários, considerando-se os cinco dias de experimento, a pesquisa do índice de confiabilidade intraclasses (ICC) variou de 0,95 a 0,99 , considerado indicador de excelente repetibilidade, de acordo com Landis e Koch ${ }^{29}$,indicando boa qualidade técnica de coleta, explicado pelo uso de avaliadores especializados.

\section{DISCUSSÃO}

Esta pesquisa dá continuidade à análise das relações entre a postura sentada e as alterações funcionais por ela induzidas ${ }^{26}$ e avaliou se diferentes mobiliários podem induzir a alterações da função respiratória. Ao analisar as posturas encontradas nos dois mobiliários estudados esperava-se que o sistema $\mathrm{A}$ induzisse a uma postura de aproximadamente $90^{\circ}$ de flexão de quadril e joelhos ${ }^{1}$, no entanto observou-se que seis participantes (40\%) - subgrupo A1 - apresentaram uma postura significativamente diferente do esperado, mas semelhante aos resultados encontrados em um estudo clássico de Grandjean e Hünting ${ }^{30}$, que relataram que os indivíduos apresentavam variações na postura sentada, de acordo com a atividade e o tempo de permanência nesta posição, sendo que em atividades de escrita deslocavam-se anteriormente no assento da cadeira, inclinando o tronco para frente e apoiando os antebraços sobre a mesa. 
Considerando a análise das variações da postura sentada, Lee et al. ${ }^{31}$ demonstraram por meio da avaliação da pressão no assento promovida por 30 participantes saudáveis, que o desequilíbrio no assento afeta a estabilização corporal negativamente, inclusive quando os indivíduos se apoiam em um lado do corpo para descanso ou realização de tarefas funcionais.

No subgrupo A2 destaca-se uma anteriorização da cabeça acentuada $\left(36^{\circ} \pm 4,56^{\circ}\right)$, ao contrário das demais posturas (subgrupo A1 e sistema B) que apresentaram valores próximos $\left(15,31^{\circ} \pm 3,16^{\circ}\right.$ e $14,6^{\circ} \pm 4,83^{\circ}$, respectivamente). Nenhuma das posturas apresentou ângulos de flexão de ombros e cotovelos dentro do recomendado na literatura pesquisada, de $25^{\circ}$ de flexão de ombros e $90^{\circ}$ de flexão de cotovelos ${ }^{32}$. Em estudo de Pheasant et al. ${ }^{33}$, foram avaliados 100 sujeitos sentados com diferentes posicionamentos da cervical. Seus resultados demonstram que de acordo com a variação da posição cervical ocorre inclusive redução da força dos ombros, o que também pode comprometer função e desempenho em tarefas.

Neste estudo, o subgrupo A2 evidenciou valores mais elevados de flexão de ombros. Acredita-se que este posicionamento, associado à anteriorização da cabeça, previamente relatada, possam induzir maior prostração das escápulas na postura sentada.

A flexão de tronco encontrada neste estudo é compatível com o encontrado na literatura ${ }^{34}$, para o uso de mesas inclinadas $\left(7^{\circ}\right)$. Os resultados no sistema $\mathrm{B}$ foram mais altos $\left(8,15^{\circ} \pm 2,38^{\circ}\right)$, o que era esperado, dada a inclinação anterior do assento da cadeira, que reduz a descarga de peso sobre as tuberosidades isquiáticas, transferindo-a parcialmente para os joelhos, favorecendo a manutenção da curvatura lombar e reduzindo a atividade da musculatura paravertebral ${ }^{17}$.

A flexão de quadril observada no sistema B se assemelha ao encontrado na chamada "postura corporal neutra”, que é de $128^{\circ}\left( \pm 7^{\circ}\right)^{35}$. Esta postura representa a posição de mínimo estresse do sistema musculoesquelético, preservando as curvaturas da coluna vertebral. Em estudo recente, de Lima e Sá Resende et al. ${ }^{36}$ demonstraram por meio da avaliação de 19 mulheres saudáveis que a distribuição do peso corporal na postura sentada tem impacto no sistema musculoesquelético e que intervenções ergonômicas ou reeducação postural podem causar redução da dor. A flexão encontrada neste mobiliário foi de $125,33^{\circ}\left( \pm 3,26^{\circ}\right)$, em seguida, o subgrupo A1, com $118,33^{\circ}( \pm 4,37)$ e, por último o subgrupo A2, com $95,77^{\circ}$ $\left( \pm 2,33^{\circ}\right)$, representando, portanto, a posição de maior sobrecarga postural. Este último grupo apresentou os valores esperados para esta flexão, assim como na flexão de joelhos, com valores de $94,33^{\circ}( \pm 10,14)$, considerando-se os estudos prévios ${ }^{32,37,38}$.

Neste parâmetro (flexão de joelho), os dados do sistema B e do subgrupo A1 foram diferentes dos demais estudos pesquisados, que variavam desde $90^{\circ}$ a $133^{\circ}$ de flexão ${ }^{1,17,30,34}$, enquanto que o encontrado neste estudo foi de $69,8^{\circ}\left( \pm 2,33^{\circ}\right)$ e $71,66^{\circ}\left( \pm 4,63^{\circ}\right)$ respectivamente.

Embora este estudo não tenha avaliado especificamente as alterações da lordose lombar, Bettany-Saltikov et al. ${ }^{18}$ relatam que a cadeira ajoelhada, com $20^{\circ}$ de inclinação possibilita a manutenção da curva lombar quando comparada à cadeira convencional.

Analisando-se, portanto, os posicionamentos encontrados em relação à flexão de quadril, e ao posicionamento de cabeça e ombros, pode-se dizer que a postura sentada encontrada no subgrupo A2 é a que induz a um posicionamento de menor eficácia biomecânica da musculatura respiratória. Em relação aos resultados da função respiratória, observou-se neste estudo que, nas posturas em que a curvatura lombar está mais próxima daquela encontrada na postura em pé, os resultados espirométricos foram melhores.

Estes resultados são similares aos encontrados por Lin et al. ${ }^{39}$, que investigaram os efeitos da postura sentada na função respiratória e na lordose lombar em quatro diferentes posturas e encontraram valores maiores na postura ortostática, em seguida em um mobiliário experimental e, por último, no mobiliário convencional. $\mathrm{O}$ foco da referida pesquisa, no entanto, foi o aumento da curva lombar, sem alteração do ângulo de flexão do quadril, ao contrário do apresentado neste estudo.

Destaca-se o fato de que a musculatura respiratória, em especial o diafragma, tem importante papel na manutenção da postura e, ao reduzirmos sua ação postural é possível favorecer sua participação na atividade respiratória. $\mathrm{Na}$ postura sentada, a musculatura posterior do tronco e da pelve é alongada a partir do posicionamento da coxa em $90^{\circ}$. Este alongamento se estende também para os pilares posteriores de inserção do diafragma, que quando encurtados impõem sobrecarga de toda musculatura envolvida com a respiração, especialmente se a pessoa sentada for obesa ou estiver grávida, pois nestes casos, existirá uma massa complementar deslocada cranialmente servindo de resistência à inspiração. Esta postura leva à utilização da postura sentada deslocada anteriormente no assento (simulando a postura semissentada), o que amplia o ângulo de flexão do quadril, liberando o abdome ${ }^{14}$. 
Este estudo indica parâmetros alterados em diferentes posturas, caracterizando sua relação com sistemas cadeiramesa diferentes (algo pouco estudado previamente), sendo possível avaliar os efeitos destas posturas na função pulmonar, e o mobiliário experimental sugerido neste estudo demonstra benefícios na função respiratória de indivíduos durante sua permanência na postura sentada.

Diferentes estudos relatam a importância do posicionamento da cabeça em relação à diminuição da expansibilidade das vias aéreas superiores ${ }^{39,40}$, uma vez que, embora a anteriorização da cabeça (flexão da coluna cervical inferior com extensão da sua porção superior) facilite a entrada de ar, ela desfavorece a relação biomecânica entre a musculatura flexora e extensora do pescoço. Porém, uma revisão sistemática conduzida por Gurani et al. ${ }^{41}$ evidenciou que a literatura é limitada, de baixa qualidade e são poucas as evidências disponíveis considerando o efeito da postura da cabeça nas dimensões das vias aéreas superiores.

O estudo de Felcar et $a 1 .{ }^{42}$ relata que esta anteriorização causa uma rotação medial de ombros, deprimindo o tórax e alterando o ritmo e a capacidade respiratória. Este posicionamento pode ser um dos fatores que justificam, ao menos parcialmente, a redução dos valores de $\mathrm{VEF}_{1}$ no subgrupo A2, que apresentou maiores ângulos de anteriorização da cabeça e de flexão de ombros. Sabendose que o $\mathrm{VEF}_{1} / \mathrm{CVF}$ é alterado sempre que um de seus parâmetros é alterado, ao reduzirmos o $\mathrm{VEF}_{1}$ a relação entre eles também é reduzida. Por outro lado, Antunes et al..$^{43}$ avaliaram 30 homens saudáveis, em posturas sentada, decúbito dorsal e ventral. Seus resultados demonstraram que o pico de fluxo expiratório é afetado de acordo com a postura corporal, sendo consideravelmente maior em indivíduos sentados em relação às outras posturas avaliadas. As diferenças na contratilidade do diafragma nas diferentes posturas em decúbito são atribuíveis às forças gravitacionais que atuam no diafragma e nas vísceras abdominais deslocadas para parte posterior ou anterior e superior do tronco. Assim, há uma resposta fisiológica do diafragma e da parede abdominal consequentes à ação destas forças, inclusive pelo aumento do retorno venoso, da força da gravidade agindo sobre as costelas e pela contenção imposta pela maca no decúbito ventral. $\mathrm{Na}$ postura sentada ou em ortostatismo a gravidade desloca o diafragma e as vísceras abdominais para uma posição inferior ${ }^{44-46}$.

De acordo com $\mathrm{West}^{47}$, o $\mathrm{FEF}_{\text {máx }}$ é volume esforço dependente e é afetado pela posição corporal. Desta forma, considerando-se que na postura ortostática a musculatura respiratória encontra-se em uma situação de vantagem mecânica em relação à posição sentada, por oposição dos membros inferiores contra o abdome e, consequentemente, deslocamento das víceras para cima, os dados encontrados neste estudo mostram-se coerentes com esta afirmação, visto que a $\mathrm{FEF}_{\text {max }}$ foi superior na posição ortostática em relação à todas as demais posições, sendo que a postura induzida pelo sistema $B$ foi a que mais se aproximou de seus valores $(8,6 \pm 2,42$ e $8,54 \pm 2,38$, respectivamente) e que este foi o único parâmetro no qual estas duas posturas (ortostática e experimental) apresentaram diferença significativa.

\section{CONCLUSÃO}

A cadeira convencional induziu dois diferentes padrões posturais, sendo que um deles apresentou ângulos articulares similares aos do sistema $\mathrm{B}$, com resultados espirométricos semelhantes. O segundo grupo apresentou ângulos corporais de acordo com o previsto e resultados espirométricos significativamente inferiores em $\mathrm{VEF}_{1}$, $\mathrm{VEF}_{1} / \mathrm{CVF}$ e $\mathrm{FEF}_{\text {max }}$. O sistema B diferiu da postura ortostática somente em $\mathrm{FEF}_{\text {máx }}$, sugerindo similaridade de situação.

Com base nos resultados aqui expostos o mobiliário experimental mostrou-se uma ferramenta viável no sentido de beneficiar a função respiratória destes indivíduos durante a permanência na postura sentada. Cadeiras para postura denominada comercialmente como semissentada estão disponíveis no mercado e a adaptação do tampo de mesas é bastante simples, viabilizando as adequações de postura.

\section{Limitações do estudo e perspectivas futuras}

Para o aprofundamento deste estudo, sugere-se considerar, inicialmente: se a permanência na postura sentada por períodos mais longos apresenta maiores variações do que as encontradas neste estudo; considerar a influência da permanência na postura sentada durante longos períodos em populações específicas, como trabalhadores e escolares; analisar, por meio de estudos de coorte, se o organismo tem capacidade de compensar estas variações sem maiores repercussões clínicas; e, por último, avaliar a adequação do mobiliário para beneficiar pessoas em condições especiais, como gestantes, obesos e pessoas com doenças pulmonares crônicas. É possível também realizar estudos com diferentes mobiliários e, em especial, com maior tempo de observação. 


\section{REFERÊNCIAS}

1. Lis AM, Black KM, Korn H, Nordin M. Association between sitting and occupational LBP. Eur Spine J. 2007;16(2):283-98. doi: 10.1007/s00586-006-0143-7

2. Claus AP, Hides JA, Moseley GL, Hodges PW. Is “ideal” sitting posture real? Measurement of spinal curves in four sitting postures. Man Ther. 2009;14(4):404-08. doi: 10.1016/j.math.2008.06.001

3. Daneshmandi H, Choobineh A, Ghaem H, Karimi M. Adverse effects of prolonged sitting behavior on the general health of office workers. J Lifestyle Med. 2017;7(2):69-75. doi: 10.15280/ jlm.2017.7.2.69

4. Sa RC, Zeman KL, Bennett WD, Prisk GK, Darquenne C. Effect of posture on regional deposition of coarse particles in the healthy human lung. J Aerosol Med Pulm Drug Deliv. 2015;28(6):423-31. doi: 10.1089/jamp.2014.1189

5. McManus AM, Ainslie PN, Green DJ, Simair RG, Smith K, Lewis $\mathrm{N}$. Impact of prolonged sitting on vascular function in young girls. Exp Physiol. 2015;100(11):1379-87. doi: 10.1113/ep085355

6. Healy GN, Winkler EAH, Owen N, Anuradha S, Dunstan DW. Replacing sitting time with standing or stepping: associations with cardio-metabolic risk biomarkers. Eur Heart J. 2015;36(39):2643-49. doi: 10.1093/eurheartj/ehv308

7. Lakerveld J, Loyen A, Schotman N, Peeters CFW, Cardon G, Van Der Ploeg HP, et al. Sitting too much: a hierarchy of socio-demographic correlates. Prev Med. 2017;101:77-83. doi: 10.1016/j.ypmed.2017.05.015

8. Biddle SJH, Bennie JA, Bauman AE, Chau JY, Dunstan D, Owen N et al. Too much sitting and all-cause mortality: is there a causal link? BMC Public Health. 2016;16:635. doi: 10.1186/s12889-016-3307-3

9. Tremblay MS, Warburton DER, Janssen I, Paterson DH, Latimer AE, Rhodes RE, et al. New Canadian physical activity guidelines: Appl Physiol Nutr Metabol. 2011;36(1):36-46. doi: 10.1139/ H11-009

10. Bull F, Biddle S, Buchner D, Ferguson R, Foster C, Fox $\mathrm{K}$, et al. Physical activity guidelines in the UK: review and recommendations. Loughborough: Loughborough University; 2010. [cited 2019 July 5]. Available from: https://mmu.rl.talis. com/items/C14E750E-74FF-8D6B-6668-2E054926EE97.html

11. Cavalcanti AG, Lima CS, de Sá RB, Reinaux CM, Braz Júnior DS, Teixeira Al, et al. Influence of posture on the ventilatory pattern and the thoraco-abdominal kinematics of patients with chronic obstructive pulmonary disease (COPD). Physiother Theory Pract. 2014;30(7):490-94. doi: 10.3109/09593985.2014.901458

12. Li C-T, Chang C-H, Huang J-H, Tsai K-H. Comparison of various sitting postures on pulmonary function, lumbar curvature, and comfort evaluations. Int J Biosci BiochemBioinforma. 2014;4(5):331.doi: 10.7763/IJBBB.2014.V4.365

13. Badr C, Elkins MR, Ellis ER. The effect of body position on maximal expiratory pressure and flow. Aus J Physiother. 2002;48(2):95-102. doi: 10.1016/S0004-9514(14)60203-8

14. Lee L-J, Chang AT, Coppieters MW, Hodges PW. Changes in sitting posture induce multiplanar changes in chest wall shape and motion with breathing. Respir Physiol Neurobiol. 2010;170(3):236-45. doi: 10.1016/j.resp.2010.01.001
15. Ricardo DR, Araújo CGSd. Body mass index: a scientific evidence-based inquiry. Arq Bras Cardiol. 2002;79:70-8. doi: 10.1590/S0066-782X2002001000007

16. Trudelle-Jackson E, Fleisher LA, Borman N, Morrow JR, Jr., Frierson GM. Lumbar spine flexion and extension extremes of motion in women of different age and racial groups: the WIN Study. Spine (Phila Pa 1976). 2010;35(16):1539-44. doi: 10.1097/BRS.0b013e3181b0c3d1

17. Makhsous M, Lin F, Bankard J, Hendrix RW, Hepler M, Press J. Biomechanical effects of sitting with adjustable ischial and lumbar support on occupational low back pain: evaluation of sitting load and back muscle activity. BMC Musculoskelet Disord. 2009;10:17. doi: 10.1186/1471-2474-10-17

18. Bettany-Saltikov J, Warren J, Jobson M. Ergonomically designed kneeling chairs are they worth it? : Comparison of sagittal lumbar curvature in two different seating postures. Stud Health Technol Inform. 2008;140:103-6. doi: 10.3233/978-1-58603-888-5-103

19. Kavak ST, Bumin G. Os efeitos da postura de pega do lápis e de diferentes modelos de mesa sobre o desempenho na caligrafia de crianças com paralisia cerebral hemiplégica. J Pediatr. 2009;85:346-52. doi: 10.2223/JPED.1914

20. Corlett EN, Bishop RP. A technique for assessing postural discomfort. Ergonomics. 1976;19(2):175-82. doi: 10.1080/00140137608931530

21. Ferreira EAG. Postura e controle postural: desenvolvimento e aplicação de método quantitativo de avaliação postural [tese]. São Paulo (SP): Universidade de São Paulo. 2005;1-114. doi: 10.11606/T.5.2006.tde-20092006-142252

22. Ferreira EAG, Duarte M, Maldonado EP, Burke TN, Marques AP. Postural assessment software (PAS/Sapo): validation and reliabiliy. Clinics. 2010;65:675-81. doi: 10.1590/S1807-59322010000700005

23. Santos MM, Silva MPC, Sanada LS, Alves CRJ. Análise postural fotogramétrica de crianças saudáveis de 7 a 10 anos: confiabilidade interexaminadores. Rev Bras Fisioter. 2009;13(4):350-5. doi: 10.1590/S1413-35552009005000047

24. Domingos-Benício NC, Gastaldi AC, Perecin JC, et al. Medidas espirométricas em pessoas eutróficas e obesas nas posições ortostática, sentada e deitada. Rev Assoc Med Bras. 2004;50(2):142-7.doi: 10.1590/S0104-42302004000200028

25. Pereira CAC. I Consenso sobre espirometria. J Pneumol. 1996[cited 2019 July 5];22(3)105-164. Available from: jornaldepneumologia.com.br/PDF/Suple_179_57_I\%20 CONSENSO\%2OBRASILEIRO\%2OSOBRE\%2OESPIROMETRIA \%201996.pdf

26. Caromano FA, Nunes Sobrinho FP. Caracterização da postura sentada em dois mobiliários diferentes e a influência no desempenho em um teste gráfico: estudos de casos. Rev Ter Ocup USP. 2001;12: 40-7.

27. Romero-Franco N, Montano-Munuera JA, Jimenez-Reyes P. Validity and reliability of a digital inclinometer to assess knee joint position sense in a closed kinetic chain. J Sport Rehabil. 2017;26(1):1-5. doi: 10.1123/jsr.2015-0138

28. Miller MR, Hankinson J, Brusasco V, Burgos F, Casaburi R, Coates A, et al. Standardisation of spirometry. Eur Respir J. 2005;26(2):319-38. doi: 10.1183/09031936.05.00034805

29. Landis JR, Koch GG. The measurement of observer agreement for categorical data. Biometrics. 1977:159-74. doi: 10.2307/2529310 
30. Grandjean E, Hunting W. Ergonomics of posture-review of various problems of standing and sitting posture. Appl Ergon. 1977;8(3):135-40. doi: 10.1016/0003-6870(77)90002-3

31. Lee DE, Seo SM, Woo HS, Won SY. Analysis of body imbalance in various writing sitting postures using sitting pressure measurement. J Phys Ther Sci. 2018;30(2):343-6. doi: 10.1589/jpts.30.343

32. Cagnie B, Danneels L, Van Tiggelen D, De Loose V, Cambier D. Individual and work related risk factors for neck pain among office workers: a cross sectional study. Eur Spine J. 2007;16(5):679-86. doi: 10.1007/s00586-006-0269-7

33. Pheasant S, Haydt R, Gottstein T, Grasso A, Lombard N, Stone B. Shoulder external rotator strength in response to various sitting postures: a controlled laboratory study. Int J Sports Phys Ther. 2018;13(1):50-7. doi: 10.26603/ijspt20180050

34. Oyewole SA, Haight JM, Freivalds A. The ergonomic design of classroom furniture/computer work station for first graders in the elementary school. Int J Ind Ergon. 2010;40(4):437-47. doi: 10.1016/j.ergon.2010.02.002

35. Congleton JJ, inventor; Jerome J. Congleton, assignee. Neutral body posture chair. In: Google Patents; 1985. United States patent 4552404. 1985 Dec 11. [cited 2019 July 5]. Available from: www.freepatentsonline.com/4552404.html

36. de Lima e Sá Resende F, Vanti C, Banchelli F, Trani JGB, Oliveira JBA, Villafañe JH, et al. The effect of global postural reeducation on body weight distribution in sitting posture and on musculoskeletal pain: a pilot study. Med Lav. 2017;108(3):187-96. doi: $10.23749 /$ mdl.v108i3.5458

37. Thompson BJ, Whitson M, Sobolewski EJ, Stock MS. Effects of age, joint angle, and test modality on strength production and functional outcomes. Int J Sports Med. 2018;39(2):124-32. doi: 10.1055/s-0043-121149

38. Babaei H, Razeghi M, Choobineh A, Pakshir H, Rajaeifard A, Rezaian J. A new method for calculating saddle seat height with an emphasis on optimal posture based on trigonometric relations. Int J Occup Saf Ergon. 2016;22(4):565-71. doi: 10.1080/10803548.2016.1191223

39. Lin F, Parthasarathy S, Taylor SJ, Pucci D, Hendrix RW, Makhsous M. Effect of different sitting postures on lung capacity, expiratory flow, and lumbar lordosis. Arch Phys Med Rehabil. 2006;87(4):504-9. doi: 10.1016/j.apmr.2005.11.031

40. Efendiyeva R, Aydemir H, Karasu H, Toygar-Memikoglu U. Pharyngeal airway space, hyoid bone position, and head posture after bimaxillary orthognathic surgery in Class III patients: long-term evaluation. Angle Orthod. 2014;84(5):773-81. doi: 10.2319/072213-534.1

41. Gurani SF, Di Carlo G, Cattaneo PM, Thorn JJ, Pinholt EM. Effect of head and tongue posture on the pharyngeal airway dimensions and morphology in three-dimensional imaging: a systematic review. J Oral Maxillofac Res. 2016;7(1):e1. doi: 10.5037/jomr.2016.7101

42. Felcar JM, Bueno IR, Massan ACS, Torezan RP, Cardoso JR. Prevalência de respiradores bucais em crianças de idade escolar. Ciên Saúde Colet. 2010;15:427-35. doi: 10.1590/S1413-81232010000200020

43. Antunes BO, de Souza HC, Gianinis HH, Passarelli-Amaro RC, Tambascio J, Gastaldi AC. Peak expiratory flow in healthy, young, non-active subjects in seated, supine, and prone postures. Physiother Theory Pract. 2016;32(6):489-93. doi: 10.3109/09593985.2016.1139646

44. Brown C, Tseng SC, Mitchell K, Roddey T. Body Position Affects ultrasonographic measurement of diaphragm contractility. Cardiopulm Phys Ther J. 2018;29(4):166-72. doi: 10.1097/cpt.0000000000000083

45. Druz WS, Sharp JT. Activity of respiratory muscles in upright and recumbent humans. J Appl Physiol Respir Environ Exerc Physiol. 1981;51(6):1552-61. doi: 10.1152/jappl.1981.51.6.1552

46. Malbrain ML, De Laet I, De Waele JJ, Sugrue M, Schachtrupp A, Duchesne J, et al. The role of abdominal compliance, the neglected parameter in critically ill patients: a consensus review of 16. Part 2: measurement techniques and management recommendations. Anaesthesiol Intensive Ther. 2014;46(5):40632. doi: 10.5603/ait.2014.0063

47. West JB. Fisiologia respiratória: princípios básicos. Porto Alegre: Artmed; 2013. ISBN-10: 8565852741 